\title{
On twinning in smectic crystals
}

\author{
V.I. Marchenko \\ Kapitza Institute for Physical Problems, RAS, Moscow, 119334 Russia
}

\begin{abstract}
It is shown that mechanical twinning in smectic crystals is possible. The structure of the boundary of twins for a small disorientation of crystallites is determined. The periodic twin structure, which should appear at the tension of the smectic layer, is proposed.
\end{abstract}

PACS numbers:

Keywords:

The energy of small deformations of a smectic crystal is given by the expression [1]

$$
\mathcal{E}=\int \frac{A}{2}\left\{\left(\partial_{z} u-\frac{\left(\partial_{\alpha} u\right)^{2}}{2}\right)^{2}+\lambda^{2}\left(\Delta_{\perp} u\right)^{2}\right\} d V,
$$

where $u$ - is the displacement of layers along $z$ axis (in the initial homogeneous undeformed state of the smectic crystal, layers lie in the $x y$ plane, $A$ - is the elastic modulus, $\lambda$ - is the length parameter, $\partial_{\alpha}$ - is the gradient vector in the $x y$ plane, and $\Delta_{\perp}=\partial_{\alpha}^{2}$.

According to Eq. (1), the undeformed state turned by small angle $\theta \ll 1$ in the $x z$ plane (in this case, $\partial_{x} u=\theta$ ) corresponds to the derivative $\partial_{z} u=\theta^{2} / 2$. Let us consider the boundary between the states $\partial_{x} u= \pm \theta(x \rightarrow \pm \infty)$ that lies in the $y z$ plane. The quantity $\partial_{z} u$ is unchanged inside the boundary. The variation equilibrium equation in the problem under consideration reduces to the form

$$
\lambda^{2} f^{\prime \prime \prime}+\frac{\theta^{2}}{2} f^{\prime}-\frac{3}{2} f^{2} f^{\prime}=0,
$$

where $f=\partial_{x} u$. The solution of this equation has the form $f=\theta \cdot \tanh (\theta x / 2 \lambda)$. The energy of the unit area of this boundary is given by the formula

$$
\sigma=2 A \lambda \theta^{3} / 3
$$

The twin structure of smectic crystals must be observed under the conditions of Helfrich instability at strains noticeably larger than the critical value (see the problem in [1, Sect. 45]: the smectic layer of thickness $L$ bounded by solid walls parallel to the smectic layer is extended along the $z$ axis). At very small tensions $\delta L>\delta L_{c}=2 \pi \lambda$, when $\delta L$ is about the smectic period, the homogeneous state becomes unstable with respect to the appearance of a periodic structure in the $x y$ plane with wave-number $k_{c}=\sqrt{\pi / \lambda L}$. At a much larger strain $\delta L \gg \delta L_{c}$, a twin structure as that schematically shown in the figure should appear.

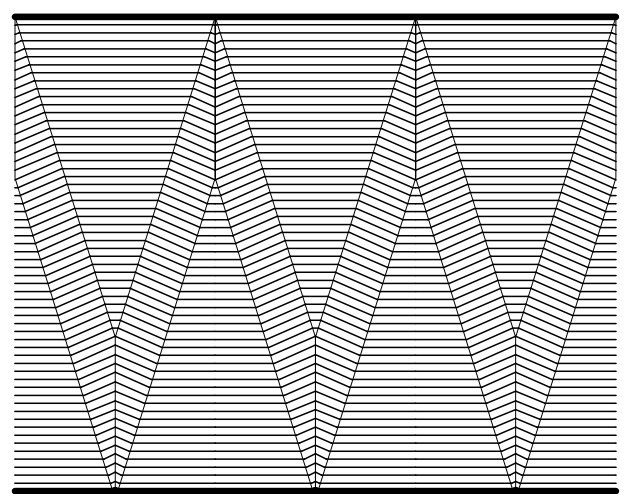

The parameters of this structure are determined by minimizing the total energy of twin boundaries $(\theta=\varepsilon$, at the vertical boundaries and $\theta=\varepsilon / 2$ at the boundaries of the triangular regions). According to geometric consideration, the angle at the vertex of a triangle is equal to $\varepsilon$, the height $H$ of the triangles is related to the structure period $d$ as $\tan (\varepsilon / 2)=d / 2 H$, and the quantity $\delta L$ is related to the parameter $\varepsilon$ as

$$
\delta L=(L-H)\left(\frac{1}{\cos \varepsilon}-1\right)
$$

The energy density of the structure proposed above is given by the expression

$$
\frac{1}{L d}\left\{2 \frac{L-H}{\cos \varepsilon} \sigma(\varepsilon)+4 \frac{H}{\cos (\varepsilon / 2)} \sigma(\varepsilon / 2)\right\} .
$$

In view of the indicated geometric relationships, at a given tension $\delta L / L$ energy (4) is a function of one parameter $\varepsilon$. When $\delta L \ll L$, angle $\varepsilon$ is small. In this case, with the use of result (3), the minimum of energy (4) is found to correspond to $\varepsilon=\sqrt{6 \delta L / L}$. In this case, $H=2 L / 3$ and

$$
d=2 \sqrt{\frac{2 L \delta L}{3}}
$$

I am grateful to E.I. Kats for stimulating discussions.

1. L.D. Landau and E.M. Lifshitz, Theory of Elasticity, Pergamon, NY (1986)

Translated by R. Tyapaev

JETP Lett. 116(4), 587 (2007) 